\title{
Management of gender dysphoria in adolescents in primary care
}

\author{
Joseph H. Bonifacio MD MPH, Catherine Maser MN, NP-Paediatric, Katie Stadelman MSW RSW, Mark Palmert MD PhD
}

- Cite as: CMAJ 2019 January 21;191:E69-75. doi: 10.1503/cmaj.180672

See related article at www.cmaj.ca/lookup/doi/10.1503/cmaj.190011

$\mathbf{A}$ dolescents with gender dysphoria present in a variety of health care settings, including primary care. Gender dysphoria is the distress experienced by an individual when their gender identity and their gender assigned at birth are discordant. Many tertiary pediatric centres across Canada and the Unites States have opened gender clinics for adolescents with gender dysphoria. ${ }^{1}$ However, high demand often exceeds the capacity of these clinics, and many youth are prevented from accessing such centres for a variety of reasons (e.g., lack of parental or physician support, geographical distance).

Primary care providers are well placed to provide critical support for youth with gender dysphoria and their caregivers and families. However, primary care providers often lack exposure to trans health issues in training, and may lack experience in managing gender dysphoria in youth. A recent Canadian national survey found that less than $50 \%$ of transgender youth felt comfortable discussing their trans-related health care needs with their family doctor. ${ }^{2}$ We provide an overview of the management of gender dysphoria in postpubertal adolescents, including practical advice on approaches to social and medical transitioning, aimed at supporting primary care practitioners in supporting youth with gender dysphoria in their practices.

We use the current Diagnostic and Statistical Manual of Mental Disorders, Fifth Edition (DSM-5) diagnostic criteria when referring to gender dysphoria. Because definitions and approaches to care have changed over the past 2 decades, we focus mainly on recent research that reflects the current diagnostic criteria, studies that apply contemporary assessment and measurement strategies, and findings that are applicable across multiple clinical settings. Our approach to gathering evidence used in this review is presented in Box 1. Box 2 defines commonly used terms.

\section{How do adolescents with gender dysphoria present in the primary care setting?}

Youth may present to either primary care or mental health care providers stating overtly that they are "transgender" and requesting a gender assessment, or they may present less overtly with a mood disorder, anxiety or depressive traits, or caregiver concern about social problems such as a change in academic

\section{Key points}

- An increasing proportion of adolescents identify as transgender.

- Small studies have shown that the mental health of adolescents with gender dysphoria improves when they receive genderaffirming care.

- Primary health care providers can support adolescents with gender dysphoria and their families by providing guidance on the timing of social transitioning, reviewing and overseeing the potential use of medical management, and connecting them with local community resources and supports.

- Hormonal suppression can delay puberty and give youth and their families more time to make decisions about transitioning.

- More research is needed to inform the optimal management of adolescents with gender dysphoria.

performance or school truancy. Concerns about gender identity may not be disclosed at initial presentation. Yet identifying and supporting adolescents with gender dysphoria is important because it may be exacerbated by mental health problems.

Depression, dysthymia or symptoms of depression affect adolescents with gender dysphoria (range: 12\%-64\%), with most studies reporting a prevalence of around 30\%. ${ }^{3-15}$ Anxiety affects $16 \%-25 \% ; 2 \%-15.8 \%$ are given a diagnosis of an eating disorder; $13.1 \%-53 \%$ have self-harmed; and 9.3\%$30 \%$ have attempted suicide..$^{5,7-16}$ Moreover, a recent study of

\section{Box 1: Evidence used in this review}

We searched MEDLINE for articles published from 1950 to 2018 using the search terms "gender dysphoria," "gender identity," and "transgender" and limited this search to studies involving youth and young adults. Of the 1337 studies identified, we excluded case reports, commentaries, editorials, letters and reviews, which left 1255 articles in English. After reviewing the titles, we selected the abstracts of articles that focused on diagnostic- or assessmentrelated issues. We retrieved the full-text versions if they were relevant to general clinical practice. The most recent articles were preferred for inclusion, particularly those that reflected the current diagnostic criteria and were applicable across multiple clinical settings. 


\section{Box 2: Definitions of commonly used terms}

- Gender assigned at birth: a person's initial assignment as male or female at birth. It is based on the child's genitalia and other visible physical sex characteristics.

- Biological or anatomic sex: the physical attributes that characterize one as male or female; usually based on genitalia at birth.

- Cisgender: individuals whose affirmed gender matches their gender assigned at birth and their physical sex characteristics.

- Gender dysphoria: distress that is caused by a discrepancy between a person's gender identity and that person's gender assigned at birth. Not all gender-variant individuals experience gender dysphoria. It is more specifically defined by the Diagnostic and Statistical Manual of Mental Disorders, Fifth Edition (DSM-5; American Psychiatric Association) as a diagnosis.

- Gender expression: the way a person communicates about gender to others through external means such as clothing, appearance or mannerisms. This communication may be conscious or subconscious and may or may not reflect gender identity or sexual orientation.

- Gender identity (or affirmed gender): a person's intrinsic sense of self as male, female or an alternate gender, reflecting a complex interplay of biological, genetic, environmental and cultural factors. It does not always correspond to biological or anatomic sex.

- Gender identity disorder: previous terminology used in the Diagnostic and Statistical Manual of Mental Disorders, Fourth Edition to describe what is now termed gender dysphoria. We use the term "gender identity disorder" when citing older literature that used that term and its definition in research studies.

- Gender nonconforming: used to describe individuals whose gender identity, role or expression differs from what is normative for their gender assigned at birth in a given culture and historical period.

- Gender role: the characteristics in personality, appearance and behaviour that, in a given culture and historical period, are designated as masculine or feminine.

- Gender variance: the behaviours, appearance or identity of people who do not conform to culturally defined norms for their gender assigned at birth.

- Female-to-male (FTM): persons assigned female gender at birth who identify as male.

- Male-to-female (MTF): persons assigned male gender at birth who identify as female.

- Transgender: used to describe individuals with an affirmed gender identity different from their gender assigned at birth and physical sex characteristics. Transgender persons are not necessarily female to male or male to female, as "transgender" can also be used to describe people whose gender identity, expression or behaviours cross or transcend culturally defined categories of gender.

- Transitioning: a process whereby individuals change their social or physical characteristics or both for the purpose of living according to their affirmed gender. Transitioning may or may not include hormonal or surgical procedures.

- Sexual orientation: the personal quality inclining persons to be romantically or physically attracted to persons of the same sex, opposite sex, both sexes or neither sex. Sexual orientation is distinct from gender identity and gender expression.

- Social transitioning: change in social role to one's affirmed gender and may include a change of name, pronoun, clothing, appearance, mannerisms, and use of gender-affirming devices such as chest binders or packers.

- Top surgery: mastectomy or chest contouring in FTM or breast implants in MTF.

- Bottom surgery: usually metoidioplasty or phalloplasty in FTM, or vaginoplasty in MTF. Bottom surgery does not necessarily involve gonadectomy.

50 consecutive referrals to a gender clinic found that referred adolescents often have a history of psychosocial and psychological vulnerability. ${ }^{17}$

\section{How common is gender dysphoria among youth, and does it persist?}

The prevalence of gender dysphoria is difficult to determine in the general population. Previously, the prevalence in adults was thought to range from $0.005 \%$ to $0.014 \%$ for people assigned male gender at birth and $0.002 \%$ to $0.003 \%$ for people assigned female gender at birth. ${ }^{18}$ These estimates are based on referrals to surgical gender reassignment clinics, however, and are therefore likely an underestimate. More recent studies suggest that $0.39 \%$ to $0.60 \%$ of adults identify as transgender, with an increasing prevalence over the past decade. ${ }^{19}$ In studies from different jurisdictions using general samples of adolescents, rates were found to be higher among youth than among adults, with $1.2 \%$ to $4.1 \%$ of adolescents reporting a gender identity different from that assigned at birth. ${ }^{6,8,20-23}$ Similar numbers of adolescents were also found to be variant in their gender expression; that is, in the way they communicated about their gender to others - either consciously or unconsciously - through external means such as clothing, personal appearance or mannerisms. .,8,20-23 $^{6}$

Not all children and youth who report gender identities different from their gender assigned at birth will experience persistent gender dysphoria. Retrospective studies suggest gender dysphoria persists from childhood into adulthood in the range of $12 \%-$ $27 \%$. $^{12}$ Prevalence studies regarding persistence are controversial, with many critics suggesting that previously reported prevalences were erroneous for a variety of reasons: loss of follow-up participants and possible misclassification as not transgender, changing criteria of gender dysphoria, and reasons for referral to specialists (e.g., youth may have been referred for not conforming to their cultural gender norms, rather than because they experienced gender dysphoria). ${ }^{24}$

The trajectory of gender dysphoria in children is different from that of gender dysphoria presenting in adolescence. Adolescents were more likely than children to continue experiencing gender dysphoria into adulthood. ${ }^{25}$ When the gender dysphoria 
of older adolescents in childhood did not persist, many were distressed by fears of teasing and shame associated with admitting they had been "wrong."11 For such reasons, it is often the goal of professionals and parents to determine for which adolescents gender dysphoria is more likely to persist into adulthood. However, a recent Canadian commentary by medical and mental health professionals cautions that such a preoccupation was a barrier for children and adolescents with gender dysphoria in accessing proper medical and mental health care. ${ }^{24}$

\section{What is the initial approach to an adolescent with gender dysphoria?}

\section{Validation and the affirming approach}

Although the consensus in the medical community in the $1960 \mathrm{~s}$ and 1970 s was to view gender variance through a disease model in which associated behaviours, expression and declared identity were deemed pathological and in need of correction, the current approach is an affirming one that does not view gender variance as pathological. ${ }^{26-29}$ The goals of the affirming approach are to destigmatize gender variance, promote self-worth, allow for opportunities to access peer support, and enable parents and other community members to create safer spaces in schools and other social environments, rather than to pathologize the adolescent's behaviour or identity. ${ }^{29,30}$

When encountering a youth with gender dysphoria, the first step for the health care provider is to validate the youth's concern or experience regarding their gender. An affirming approach is not directive and practitioners should avoid influencing the adolescent to move down a path they would not have chosen for themselves. Sometimes youth who have just started to question their gender assigned at birth may feel pressure either to transition or not to transition from their family, peers or community. Lack of caregiver or parental support can make validation and affirmation difficult (but perhaps even more important). Ideally, the approach to youth with gender dysphoria revolves around collaborative decision-making among the youth, family or guardians, and care providers. The youth's voice is always paramount. The findings of a recent report indicated that youth who make gender decisions within a supportive environment fare better than those without family support, and care providers should work to solidify that support whenever possible. ${ }^{31}$

Ways to incorporate the affirming approach into clinical practice, including approaches to social, medical and surgical management, have been described in depth elsewhere. ${ }^{32}$ It is helpful if all practice staff are trained, before encountering youth with gender dysphoria or gender variance, to use gender-affirming language and refrain from stigmatizing youth with gender dysphoria. For example, forms and documents can include options other than "male" and "female" for gender designation. Consider making signage, including that for washrooms, gender neutral.

\section{Considering the diagnosis}

The diagnosis of gender dysphoria is not based on laboratory or imaging tests. Semistructured interviews can help in making the diagnosis, and many questionnaires have been developed and may be used to provide additional information in clarifying the diagnosis and guiding effective management. ${ }^{33,34}$ While bearing in mind the DSM-5 criteria for gender dysphoria, it is also important to recognize that there may be differences from the expected narrative. For example, the DSM-5 diagnostic criteria are based on identifying symptoms that have been present for at least 6 months; therefore, symptoms may or may not have been present in childhood. Furthermore, the diagnosis of gender dysphoria requires report of substantial distress or problems with functioning, but youth who have been living in their affirmed gender may not necessarily have experienced such distress, and therefore may not meet this criterion.

The World Professional Association for Transgender Health Standards of Care recommend regimens for hormone therapy in adolescents with gender dysphoria that are substantially different from those used in adults; these are adapted to account for the somatic, emotional and mental development that occurs throughout adolescence. ${ }^{35}$ Because there may be concurrent mental illness or psychosocial vulnerabilities, these standards of care recommend mental health assessment for both gender dysphoria and other concurrent mental health concerns, and advises counselling, supportive psychotherapy or appropriate psychoactive medications. ${ }^{35}$

Some see the association's requirement for a mental health assessment as a barrier to care, especially in situations where resources are limited. We argue, however, that even if health practitioners feel unqualified to make a gender assessment, they should still address other pressing mental or physical health issues while a youth waits for a gender assessment. For example, if a provider encounters an adolescent who appears to have gender dysphoria and symptoms of anxiety, it would be prudent for that practitioner to manage the patient's anxiety while waiting for the gender assessment. Resources for youth and their families are presented in Box 3.

It also seems reasonable to us that such assessments not be limited to psychiatrists, psychologists or social workers; rather, experienced family practice, pediatric and adolescent medicine providers should be able to conduct an evaluation of a child or youth with gender dysphoria. Providers must also be prepared to make ongoing assessments if new concerns arise.

\section{How should youth be supported to transition?}

Suggestions regarding social, medical or surgical transitioning are unique and approaches should be tailored to the individual adolescent. We do not offer guidance on surgical transitioning other than to present some limited evidence.

\section{Social transition}

Social transitioning may include changing a name, choosing a preferred pronoun, altering clothing or hairstyle, and adopting new activities and mannerisms. Youth may change only a few of these or many of them, gradually or all at once. Each adolescent's social transition will be different and timing of transitioning varies. In our clinical experience, most youth have already socially transitioned at home or at school or both before presenting to our service, with 
Box 3: Resources for children, caregivers and clinicians

\section{For parents:}

- Gender Creative Kids: http://gendercreativekids.ca/

- Families in Transition: https://ctys.org/wp-content/uploads/ CTYS-FIT-Families-in-Transition-Guide-2nd-edition.pdf

- Family Acceptance Project Family Acceptance Project (US): Online resources for families of lesbian, gay, bisexual and transgender youth: http://familyproject.sfsu.edu/

For clinicians:

- American Psychiatric Association: https://psychiatry.org/ patients-families/gender-dysphoria/what-is-gender-dysphoria

- Endocrine Society Guideline: https://academic.oup.com/jcem/ article-lookup/doi/10.1210/jc.2017-01658

- Human Rights Campaign: https://hrc.org/resources/supporting -caring-for-transgender-children

- Trans Care BC: http://phsa.ca/our-services/programs-services/ trans-care-bc

- Center for Excellence of Transgender Health: http://transhealth. ucsf.edu

- World Professional Association for Transgender Health: http:// wpath.org/

many already using a preferred name and pronoun. A study conducted at 1 clinic found that, from 2005 to 2009, the proportion of children who had socially transitioned at the time of referral increased from $8.9 \%$ (16 out of 180 ) to $33.3 \%$ (60 out of 180 ). ${ }^{36}$ Such trends may reflect studies that indirectly support the use of the affirming approach in adolescents with gender dysphoria.

A recent study examining self-reported depression, anxiety and self-worth in transgender children aged 9-14 years who had socially transitioned compared with control groups of age-matched peers and siblings found that depression and self-worth were not significantly different between groups. ${ }^{37}$ Despite these findings, parents of these transgender youth still reported higher levels of anxiety among their children, compared with children from the control group.The same research group also found that transgender children who had socially transitioned and who were supported in their gender identity had developmentally normative levels of depression and only minimal elevations in anxiety. ${ }^{38}$ Together, these studies suggest psychopathology is not inevitable within this group and suggest benefits of social transition. Although these findings are encouraging, they do require replication.

We find that working with caregivers to support them in what to expect is important. Frequently, caregivers ask about optimal timing of social transitioning as caregivers often realize the importance for youth's overall well-being when they live in their affirmed gender, yet may feel concerned about their safety. Primary care practitioners can facilitate discussion with youth and their caregivers, assisting them in balancing safety concerns while considering the distress and other mental health consequences that arise from not being able to transition socially.

\section{Hormonal suppression}

The Endocrine Society's clinical practice guidelines recommend hormonal suppression for adolescents with gender dysphoria because many experience extreme discomfort with their changing bodies during puberty. ${ }^{39,40}$ For example, youth assigned female gender at birth may be distressed by ongoing breast development or vaginal bleeding and those assigned male may find erections and facial hair disturbing. Many gender-variant youth may experience the physical changes of puberty as traumatic, with further negative consequences for mental health. Medications that suppress puberty - hormone blockers - allow youth time to explore their gender identity and expression without having to worry about ongoing pubertal changes and development of secondary sexual characteristics that may be psychologically disturbing and undesired. This can also allow families time to access resources and support and adjust to changes within the family; it can also be a time in which to explore next steps. ${ }^{1}$

The Endocrine Society recommends that pubertal suppression should not occur before puberty. ${ }^{40}$ Suppression may occur at pubertal stage Tanner II, which occurs on average at age 10.5 years in individuals assigned female gender at birth and 11.5 years in those assigned male gender at birth. This represents the stage at which secondary sexual characteristics begin to appear. The society recommends pubertal suppression only after Tanner II, as beginning puberty often solidifies the diagnosis of gender dysphoria. Some youth find that their dysphoria abates as puberty starts, making it important to allow initial pubertal changes to occur. On the other hand, some youth may find their gender dysphoria increases with puberty, corroborating their need for further care.

A mainstay of reversible hormone blockade for youth with gender dysphoria is the administration of gonadotropinreleasing hormone $(\mathrm{GnRH})$ agonists. ${ }^{41}$ These agents provide a nonpulsatile, continuous release of a $\mathrm{GnRH}$ analogue that desensitizes the $\mathrm{GnRH}$ receptors on the pituitary gland, leading to the inhibition of secretion of luteinizing hormone and folliculestimulating hormone, reduced secretion of gonadal sex steroids, and a prepubertal physiologic state. Because GnRH agonists inhibit secretion of luteinizing hormone and follicule-stimulating hormone, they are particularly useful in the treatment of youth who have entered puberty.

Use of alternative methods to suppress hormonal secretion or effect is based on provider and patient preference; these include anti-androgens such as spironolactone or cyproterone acetate for youth assigned male gender at birth, or continuous oral contraceptive pills or progestins such as medroxyprogesterone acetate for those assigned female gender at birth. Medical regimens can be found in the clinical guideline from the Endocrine Society. ${ }^{40}$ Medications to suppress puberty according to the gender assigned at birth prevent further development of secondary sex characteristics and can limit menses or penile erections when desired, even when puberty is advanced.

One concern with using $\mathrm{GnRH}$ agonists is their potential effects on bone health. Available data among adolescents who have been administered $\mathrm{GnRH}$ agonists indicate that the bone mineral density of treated youth decreased during therapy when calculated as an age-based $z$ score. ${ }^{41-43} \mathrm{~A}$ recent study of 34 youth concluded the bone mineral density of youth administered $\mathrm{GnRH}$ agonists was below their pretreatment potential, and that either 
attainment of peak bone mass had been delayed or peak bone mass itself was reduced. ${ }^{42}$ However, an earlier study found that the subsequent administration of cross-sex hormones substantially increased bone density back to age-appropriate levels both in people with affirmed male and female gender. This study also noted no statistically significant changes in lipid or carbohydrate metabolism. ${ }^{41}$ Nonetheless, for youth receiving treatment with $\mathrm{GnRH}$ agonists, appropriate treatment with vitamin D and advice to optimize calcium intake and participate in weight-bearing exercise to support bone health would seem wise.

In one study, the mental health effects of GnRH agonists were assessed by measuring the psychological status (gender dysphoria, body image, global functioning, depression, anxiety and emotional and behavioural problems), social and educational or professional functioning, quality of life and happiness among 70 youth on treatment. ${ }^{27}$ These variables were measured twice: first before suppression of puberty and then again afterward, just before the patient commenced gender-affirming hormones. After pubertal suppression, most behavioural and emotional problems and depressive symptoms decreased while general functioning improved. Feelings of anxiety and anger persisted while the degree of gender dysphoria did not change.

\section{Gender-affirming hormone therapy}

The Endocrine Society's 2009 guideline recommends that gender-affirming hormones may be administered at age 16 years or older to achieve physical gender transition. Many clinicians, however, face difficulty with such strict age criteria, as many younger pubertal youth have been living in their affirmed gender for several years, and prolonged delay of gender-affirming hormone therapy could potentially lead to negative mental health outcomes. Indeed, in the recent update of its 2009 clinical practice guideline, the Endocrine Society recognizes that some youth may benefit from earlier administration of gender-affirming hormones (i.e., between age 13.5 and 16 yr). ${ }^{40}$ Many youth understandably express the desire to go through puberty in their affirmed gender at similar ages to their peers, which would necessitate starting gender-affirming hormones at even younger ages than these new recommendations. However, some commentators have cautioned about unintended consequences of younger and younger youth initiating partially irreversible changes to their bodies. ${ }^{44,45}$ For all these reasons, age criteria for starting cross-sex hormones will likely continue to be an area of debate and uncertainty.

A small body of evidence suggests that both cross-sex hormones and gender-affirming surgery benefit adolescents with gender dysphoria. The only study looking at effects of genderaffirming hormones and surgery in adolescents investigated the mental health of 55 transgender youth from the previously mentioned Dutch study. ${ }^{46}$ Improvements in psychological functioning were positively correlated with subjective well-being postsurgery. Moreover, the well-being of these young adults was similar to or better than same-age young adults from the general population.

A 2015 study of 101 youth from the US currently taking gender-affirming hormones examined their physiologic effects. ${ }^{15}$ After extensive and frequent laboratory examination, the authors showed no correlation between use of hormone therapy and clinically important metabolic changes. ${ }^{15}$ Delay in treatment with cross-sex hormones, if associated with GnRH agonist treatment for more than a few years, may place youth at higher risk of osteopenia or osteoporosis through a more prolonged impact on bone mineral density. ${ }^{47}$

Although there is concern regarding administering partially irreversible medications, we would emphasize that disadvantages conferred by the potential adverse effects of $\mathrm{GnRH}$ agonists and concerns about the long-term effects of treating with crosssex hormones must be weighed against the negative mental health consequences of delaying their administration and the social difficulty for youth who go through puberty much later than their peers.

\section{Fertility preservation}

Clinicians face additional issues with the provision of earlier gender-affirming therapy. Although the effects of hormone blockers are reversible, commencing them at earlier ages may preclude adolescents from pursuing fertility preservation, especially if medical management progresses from blockers to administration of cross-sex hormones at an earlier age, preventing sufficient endogenous pubertal development to attain reproductive capacity. However, even with current ages of medication administration, a recent study found that very few youth with gender dysphoria opted for fertility preservation procedures such as sperm or egg harvesting. ${ }^{48,49}$ More research is required to understand why this is the case. Although it may be important to delay medical therapy temporarily to allow for fertility preservation, decisions regarding future fertility should not preclude care to address gender dysphoria.

\section{What are the gaps in knowledge?}

The care of transgender youth is still a relatively young field, with a limited but growing body of research to inform care. Most evidence has come from studies on a small group of patients in the Netherlands. Research from other centres on the effects of social, medical and surgical management, and from specialized groups - such as youth mental health organizations that have experience working with people who have gender identity concerns would add to the evidence base and may help to refine our understanding of the optimal care for youth with gender dysphoria.

Recent studies have suggested a change in the sex ratio of adolescents presenting to transgender clinics, with many more youth who are assigned female gender at birth seeking care than those assigned male. ${ }^{50-52}$ One clinic reported that more than $85 \%$ of patients seeking to transition were assigned female gender at birth. ${ }^{20} \mathrm{~A}$ recent Canadian national survey also found a higher proportion of people assigned female gender at birth seeking to transition. ${ }^{2}$ The reason for this phenomenon is not known and is likely multifactorial, but one hypothesis could be that there is greater societal acceptance of transgender men than women.

Some studies have suggested a link between gender dysphoria and autism-spectrum disorder with elevated rates of the disorder found in those referred to gender clinics, and vice versa. 
The only study that used diagnostic interviews found $7.8 \%$ of those referred to a gender clinic met criteria for autism-spectrum disorder, whereas $4.7 \%$ of those eventually given a diagnosis of gender identity disorder met criteria for autism-spectrum disorder. ${ }^{53}$ Earlier this year, a consortium of experts on gender dysphoria and autism-spectrum disorder, from various backgrounds, such as developmental pediatrics, pediatric endocrinology, psychiatry and psychology, suggested screening for autismspectrum disorder in gender dysphoria, and vice versa, and an extended diagnostic period including overlap of both treatment and ongoing assessment. ${ }^{54}$ However, methodological issues concerning the proper diagnosis of gender identity disorder (v. gender variance) and autism-spectrum disorder in these studies have led others to conclude that such rates are not necessarily elevated but only emphasize an association between the 2 conditions. ${ }^{55}$ Taken together, the basis of the increased prevalence of autism-spectrum disorder among those with gender dysphoria, and whether broad screening is warranted, remain unclear.

Studies have also suggested that youth with gender dysphoria are overrepresented in at-risk populations. A recent study found that adopted children were referred to a gender dysphoria clinic more than would be expected, based on the percentage of adopted children in the state and in the US as a whole..$^{56}$ It is unclear whether adopted youth have a higher true risk of gender dysphoria or whether the finding stems from ascertainment bias. Youth with gender dysphoria are also overrepresented in the homeless or street-involved population, but this association is likely secondary to parental and family rejection, which could lead to homelessness. ${ }^{57}$

The trend toward earlier intervention to support transition among youth with gender dysphoria is not limited to social transition and medical management; anecdotally, more youth are also requesting surgical management at younger ages, such as top surgery in people assigned female gender at birth. It will be important to obtain outcome data related to these interventions to inform future decision-making.

A population that has not been captured fully in the above research is the growing number of youth who identify with genders outside of the male-female binary. ${ }^{2,15}$ In a national Canadian study of transgender youth, $41 \%$ of youth respondents identified as nonbinary. ${ }^{58}$ A Finnish study of a general sample of adolescents found that many youth also did not identify as either male or female, instead identifying as, for example, "agender."13 From our own experience, many youth now describe their gender as nonbinary, with many people assigned female gender at birth identifying as "transmasculine," or as a gender identity that is "not male" but also not female. Further research is needed to establish the needs of this population.

Our clinic populations in Toronto are not representative of the ethnic diversity of the city's general population. The likely underrepresentation of minority ethnic and Indigenous youth is one area deserving of much further attention. ${ }^{52}$

Practically speaking, the intersection of multiple identities (e.g., ethnicity, race, socioeconomic privilege, geographical location), medical and psychological comorbidities, and transgender identity will affect the resources suggested to youths and their families. Specialized youth groups and mental health organizations may have experience working with these unique populations. However, accessing optimal individualized care may be difficult for certain populations, making it important that generalists are supported to increase their capacity to care for youth with gender dysphoria and to liaise with other professionals to support families.

\section{Conclusion}

An increasing number of adolescents seeking care for gender dysphoria suggests that more primary care providers will encounter such youth in clinical practice and should be familiar with the key aspects of their management. Box 3 provides useful resources for practitioners, youth and caregivers. Transgender care has benefited greatly from detailed guidelines, but practice is hampered by knowledge gaps. Best management will continue to evolve as new research emerges. However, the hallmark of care will remain a thoughtful, affirming, well-reasoned individualized approach that attempts to maximize support for this vulnerable population, as youth and their caregivers make complex and difficult decisions.

\section{References}

1. Bonifacio HJ, Rosenthal SM. Gender variance and dysphoria in children and adolescents. Pediatr Clin North Am 2015;62:1001-16.

2. Veale J, Saewyc E, Frohard-Dourlent H, et al. Being safe, being me: results of the Canadian Trans Youth Health Survey. Vancouver: Stigma and Resilience Among Vulnerable Youth Centre - University of British Columbia; 2015.

3. Veale JF, Watson RJ, Peter T, et al. Mental health disparities among Canadian transgender youth. J Adolesc Health 2017;60:44-9.

4. de Vries AL, Doreleijers TA, Steensma TD, et al. Psychiatric comorbidity in gender dysphoria adolescents. J Child Psychol Psychiatry 2011;52:1195-202.

5. Spack NP, Edwards-Leeper L, Feldman HA, et al. Children and adolescents with gender identity disorder referred to a pediatric medical center. Pediatrics 2012;129:418-25.

6. Shields JP, Cohen R, Glassman JR, et al. Estimating population size and demographic characteristics of lesbian, gay, bisexual, and transgender youth in middle school. J Adolesc Health 2013;52:248-50.

7. Khatchadourian K, Amed S, Metzger DL. Clinical management of youth with gender dysphoria in Vancouver. J Pediatr 2014;164:906-11.

8. Clark TC, Lucassen MF, Bullen P, et al. The health and well-being of transgender high school students: results from the New Zealand Adolescent Health Survey (Youth'12). J Adolesc Health 2014;55:93-9.

9. Reisner SL, Vetters R, Leclerc M, et al. Mental health of transgender youth in care at an adolescent urban community health center: a matched retrospective cohort study. J Adolesc Health 2015;56:274-9.

10. Reisner SL, Biello KB, White Hughto JM, et al. Psychiatric diagnoses and comorbidities in a diverse, multicity cohort of young transgender women. JAMA Pediatr 2016;170:481-6.

11. Chen M, Fuqua J, Eugster EA. Characteristics of referrals for gender dysphoria over a 13-year period. J Adolesc Health 2016;58:369-71.

12. Holt V, Skagerberg E, Dunsford M. Young people with features of gender dysphoria: demographics and associated difficulties. Clin Child Psychol Psychiatry 2016;21:108-18.

13. Kaltiala-Heino R, Sumia M, Työläjärvi M, et al. Two years of gender identity service for minors: overrepresentation of natal girls with severe problems in adolescent development. Child Adolesc Psychiatry Ment Health 2015;9:9.

14. Diemer EW, Grant JD, Munn-Chernoff MA, et al. Gender identity, sexual orientation, and eating-related pathology in a national sample of college students. J Adolesc Health 2015;57:144-9.

15. Olson J, Schrager SM, Belzer M, et al. Baseline physiologic and psychosocial characteristics of transgender youth seeking care for gender dysphoria. J Adolesc Health 2015;57:374-80.

16. Arcelus J, Claes L, Witcomb GL et al. Risk factors for non-suicidal self-injury among trans youth. J Sex Med 2016;13:402-12. 
17. Bechard M, VanderLaan DP, Wood H, et al. Psychosocial and psychological vulnerability in adolescents with gender dysphoria: a "proof of principle" study. J Sex Marital Ther 2017;43:678-88.

18. Diagnostic and statistical manual of mental disorders, 5th edition (DSM-5). Arlington (VA): American Psychiatric Association; 2013

19. Flores AR, Herman JL, GJ Gates, et al. How many adults identify as transgender in the United States. Los Angeles: UCLA-Williams Institute; 2016.

20. Sumia M, Lindberg N, Työläjärvi $M$, et al. Current and recalled childhood gender identity in community youth in comparison to referred adolescents seeking sex reassignment. J Adolesc 2017;56:34-9.

21. Swanbrow Becker MA, Nemeth Roberts SF, Ritts SM, et al. Supporting transgender college students: implications for clinical intervention and campus prevention. J Coll Stud Psychother 2017;31:155-76.

22. Becker I, Ravens-Sieberer U, Ottová-Jordan V, et al. Prevalence of adolescent gender experiences and gender expression in Germany. J Adolesc Health 2017;61:83-90.

23. Eisenberg ME, Gower AL, McMorris BJ, et al. Risk and protective factors in the lives of transgender/gender nonconforming adolescents. J Adolesc Health 2017;61:521-6.

24. Newhook JT, Pyne J, Winters K, et al. A critical commentary on follow-up studies and "desistance" theories about transgender and gender-nonconforming children. Int J Transgenderism 2018;19:212-24.

25. de Vries ALC, Steensma TD, Doreleijers TA, et al. Puberty suppression in adolescents with gender identity disorder: a prospective follow-up study. J Sex Med 2011;8:2276-83.

26. Green R. Diagnosis and treatment of gender identity disorders during childhood. Arch Sex Behav 1971;1:167-73.

27. Rekers GA, Lovaas OI. Behavioral treatment of deviant sex-role behaviors in a male child1. J Appl Behav Anal 1974;7:173-90.

28. Stein F, Remley K. AAP statement in support of transgender children, adolescents and young adults. Itasca (IL): American Academy of Pediatrics; 2017

29. Newhook JT, Winters K, Pyne J, et al. "Teach your parents and providers well." Call for refocus on the health of trans and gender-diverse children. Can Fam Physician 2018;64:332-5.

30. Saeger K. Finding our way: guiding a young transgender child. J GLBT Fam Stud 2006;2:207-45.

31. Travers R, Bauer G, Pyne J, et al. Impacts of strong parental support for trans youth: a report prepared for Children's Aid Society of Toronto and Delisle Youth Services. In Trans PULSE Project. TransPulse; 2012.

32. Menvielle E. A comprehensive program for children with gender variant behaviors and gender identity disorders. J Homosex 2012;59:357-68.

33. Singh D, Deogracias JJ, Johnson LL, et al. The gender identity/gender dysphoria questionnaire for adolescents and adults: further validity evidence. J Sex Res 2010;47:49-58.

34. Wallien MSC, Quilty LC, Steensma TD, et al. Cross-national replication of the gender identity interview for children. J Pers Assess 2009;91:545-52.

35. Coleman E, Bockting W, Botzer M, et al. Standards of care for the health of transsexual, transgender, and gender-nonconforming people, version 7. Int J Transgenderism 2012;13:165-232.

36. Steensma TD, Cohen-Kettenis PT. Gender transitioning before puberty? Arch Sex Behav 2011;40:649-50.

37. Durwood L, McLaughlin KA, Olson KR. Mental health and self-worth in socially transitioned transgender youth. J Am Acad Child Adolesc Psychiatry 2017;56:116123.e2.

38. Olson KR, Durwood L, DeMeules M, et al. Mental health of transgender children who are supported in their identities. Pediatrics 2016;137:e20153223.

39. Hembree WC, Cohen-Kittenis P, Delemarre-van de Waal H, et al. Endocrine treatment of transsexual persons: an Endocrine Society clinical practice guideline. J Clin Endocrinol Metab 2009;94:3132-54.

40. Hembree WC, Cohen-Kettenis PT, Gooren L, et al. Endocrine treatment of gender-dysphoric/gender-incongruent persons: an Endocrine Society* clinical practice guideline. J Clin Endocrinol Metab 2017;102:3869-903.

41. Delemarre-van de Waal HA, Cohen-Kettenis PT. Clinical management of gender identity disorder in adolescents: a protocol on psychological and paediatric endocrinology aspects. Eur J Endocrinol 2006;155(Suppl 1): S131-7.
42. Klink D, Caris M, Heijboer A, et al. Bone mass in young adulthood following gonadotropin-releasing hormone analog treatment and cross-sex hormone treatment in adolescents with gender dysphoria. J Clin Endocrinol Metab 2015;100:E270-5.

43. Rosenthal SM. Transgender youth: current concepts. Ann Pediatr Endocrinol Metab 2016;21:185-92.

44. Vrouenraets LJ, Fredriks AM, Hannema SE, et al. Early medical treatment of children and adolescents with gender dysphoria: an empirical ethical study. J Adolesc Health 2015;57:367-73.

45. Chen D, Edwards-Leeper L, Stancin T, et al. Advancing the practice of pediatric psychology with transgender youth: state of the science, ongoing controversies, and future directions. Clin Pract Pediatr Psychol 2018;6:73-83

46. de Vries AL, McGuire JK, Steensma TD, et al. Young adult psychological outcome after puberty suppression and gender reassignment. Pediatrics 2014;134:696-704.

47. Olson-Kennedy J, Rosenthal SM, Hastings J, et al. Health considerations for gender non-conforming children and transgender adolescents. San Francisco: Center of Excellence for Transgender Health University of California, San Francisco; 2018 Available: http://transhealth.ucsf.edu/trans?page=guidelines-youth (accessed 2018 May 2).

48. Nahata L, Tishelman AC, Caltabellotta NM, et al. Low fertility preservation utilization among transgender youth. J Adolesc Health 2017;61:40-4.

49. Chen $D$, Simons L, Johnson EK, et al. Fertility preservation for transgender adolescents. J Adolesc Health 2017;61:120-3.

50. Aitken M, Steensma TD, Blanchard R, et al. Evidence for an altered sex ratio in clinic-referred adolescents with gender dysphoria. J Sex Med 2015;12:756-63.

51. Zucker KJ. Epidemiology of gender dysphoria and transgender identity. Sex Health 2017;14:404-11.

52. Chiniara LN, Bonifacio HJ, Palmert MR. Characteristics of adolescents referred to a gender clinic: are youth seen now different from those in initial reports? Horm Res Paediatr 2018;89:434-441.

53. de Vries AL, Noens IL, Cohen-Kettenis PT, et al. Autism spectrum disorders in gender dysphoric children and adolescents. J Autism Dev Disord 2010;40:930-6.

54. Strang JF, Meagher H, Kenworthy L, et al. Initial clinical guidelines for cooccurring autism spectrum disorder and gender dysphoria or incongruence in adolescents. J Clin Child Adolesc Psychol 2018;47:105-15

55. Turban JL, van Schalkwyk G. "Gender dysphoria" and autism spectrum disorder: Is the link real? J Am Acad Child Adolesc Psychiatry 2018;57:8-9.e2.

56. Shumer DE, Abrha A, Feldman HA, et al. Overrepresentation of adopted adolescents at a hospital-based gender dysphoria clinic. Transgender Health 2017;2:76-9.

57. Corliss HL, Goodenow CS, Nichols L, et al. High burden of homelessness among sexual-minority adolescents: findings from a representative Massachusetts high school sample. Am J Public Health 2011;101:1683-9.

58. Clark BA, Veale JF, Townsend M, et al. Non-binary youth: access to gender-affirming primary health care. Int J Transgenderism 2018;19:158-69.

\section{Competing interests: None declared.}

This article has been peer reviewed.

Affiliations: Department of Pediatrics (Bonifacio), St. Michael's Hospital; Departments of Pediatric Endocrinology (Palmert) and of Adolescent Medicine (Maser, Stadelman), The Hospital for Sick Children; Lawrence S. Bloomberg Faculty of Nursing (Maser), University of Toronto, Toronto, Ont.

Contributors: All of the authors contributed to the conception and design of the work, and the acquisition, analysis, and interpretation of data. All of the authors drafted the manuscript, revised it critically for important intellectual content, gave final approval of the version to be published and agreed to be accountable for all aspects of the work.

Correspondence to: Joseph Bonifacio, bonifacioh@smh.ca; or Mark Palmert, mark.palmert@sickkids.ca 